\title{
Restrição de Tempo Afeta na Experiência de Fluxo e no Ensino de Literatura? Estudo Experimental e Análises no Kahoot!
}

\author{
Deise de Lacerda Paiva, Universidade de São Paulo, deisepaiva@usp.br, \\ ORCID: 0000-0001-9283-6830 \\ Geiser Chalco Challco, Universidade Federal de Alagoas, geiser@alumni.usp.br, \\ ORCID: 0000-0003-4163-4803 \\ Seiji Isotani, Universidade de São Paulo, sisotani@icmc.usp.br, \\ ORCID: 0000-0003-1574-0784 \\ Ig Ibert Bittencourt, Universidade Federal de Alagoas, ig.iber@ic.ufal.br, \\ ORCID: 0000-0001-5676-2280
}

\begin{abstract}
Resumo
A gamificação tem sido utilizada em contextos educacionais para melhorar a motivação e o engajamento dos alunos. Embora existam diversos estudos que investigam esse fenômeno, há um desconhecimento sobre o efeito de cada um dos elementos do jogo. Portanto, realizamos um estudo empírico para compreender os efeitos do elementojogo "restrição de tempo" na disciplina de Literatura do $1^{o}$ ano do ensino médio de uma escola privada brasileira. $O$ estudo centrou-se na análise do estado de fluxo dos alunos e na sua aprendizagem, utilizando o quiz do Kahoot!. Observou-se que, comparando o cenário com restrição de tempo e sem restrição de tempo, não houve diferença significativa no estado de fluxo, mas a aprendizagem no cenário sem limite de tempo foi melhor.
\end{abstract}

Palavras-chave: gamificação, elemento-jogo; ensino de literatura

\section{Does time constraint affect the flow and learning experience in literature? Experimental study and analysis on Kahoot!}

\begin{abstract}
Gamification has been used in educational contexts to improve the students' motivation and engagement. Although there are several studies that investigate this phenomenon, there is a lack of understanding about the effect of each one of game elements. Therefore, we conducted an empirical study to understand the effects of the gameelement "time limit" in the Literature course for the 1st year of upper secondary education of a private Brazilian school. The study focused on the analysis of the students' flow state and their learning, using Kahoot! quizzes. It was observed that, comparing the scenario with time limit and without time limit, there was not significant difference in the flow state, but the learning in the scenario without time limit was significantly better than in the scenario with time limit.
\end{abstract}

Keywords: gamification; game-element; literature.

\section{Introdução}

Parece haver um consenso, entre os estudiosos do tema, de que o letramento literário ${ }^{1}$ é a fonte e principal função do ensino de literatura na escola(Cosson, 2006). Isso significa

\footnotetext{
${ }^{1}$ Cabe aqui esclarecer que, na atualidade, "alfabetização" e "letramento" são considerados processos simultâneos, "a conveniência, porém, de conservar os dois termos parece (...) estar em que, embora designem processos interdependentes, indissociáveis e simultâneos, são processos de natureza
} 
que caberia à escola proporcionar ao aluno a habilidade de construir sentidos próprios da linguagem literária. Aliado a isso, por ser uma criação artística por excelência, o contato com o texto literário na escola deve contribuir para a fabulação, considerada uma necessidade humana(Formiga e Inácio, 2017); sem mencionar que a literatura abre lugar para outros saberes, como o histórico, geográfico, social, técnico, botânico, antropológico etc. (Silva, Rodrigues e Leal, 2019). No entanto, observa-se um crescente desinteresse dos alunos pelo estudo da literatura, o que, segundo os estudiosos, é atribuído a diversos fatores, como a preferência do ensino da literatura pela perspectiva histórica e pelo posicionamento idealista, dividindo-a por nacionalidades(Formiga e Inácio, 2017).

De modo geral, os recursos educacionais tradicionais adotados pelas instituições de ensino não despertam o interesse de alunos das novas gerações. Por isso, para reverter esse cenário, as escolas vêm adotando diferentes estratégias pedagógicas para promover a aproximação dos estudantes ao texto literário. Um exemplo de tais estratégias é a aplicação da gamificação, que tem sido utilizada principalmente para contribuir com o aumento da motivação, do engajamento e do aprendizado dos alunos em atividades educacionais.

Diferentes estudos(Challco et al., 2016; Oliveira et al., 2021; Santos et al., 2018), demonstraram que o planejamento adequado da atividade educacional, que contemple, inclusive, uma avaliação dos perfis dos estudantes, aliado à inclusão de elementos da gamificação, como conquistas, desafios, progressos, entre outros, favorecem o alcance de um estado conhecido por "flow" (fluxo), uma espécie de imersão na realização em dada atividade, tornando, assim, a aprendizagem mais atrativa e prazerosa.

Embora exista um interesse crescente pelo tema gamificação e suas aplicações, a maioria dos estudos estão relacionados às questões de engajamento e de motivação de modo geral. Por isso, é relevante uma maior compreensão dos efeitos que cada elemento pode causar nos cenários de aprendizagem(Faiella e Ricciardi, 2015). Mesmo que a literatura ainda não tenha estabelecido qual é a unidade de elemento de design de jogo mínima que deve ser estudada(Mora et al., 2017), a maioria dos estudos de gamificação costuma usar dois ou mais elementos(Dicheva et al., 2015). No entanto, esses elementos são, em sua maioria, componentes (isto é, pontos, crachás, classificação). Neste estudo, decidimos por empregar como unidade mínima de estudo uma dinâmica de jogo e, assim, explorarmos os efeitos da dinâmica "restrição de tempo para completar uma atividade." A dinâmica foi implementada pelas mecânicas "desafio", "competição" e "feedback", e pelos componentes "pontos" e "pontuações" (classificação) na plataformaKahoot!.

O Kahoot! é uma plataforma de aprendizagem baseada em jogos, que incluiquiz, falso ou verdadeiro, múltipla escolha, quebra-cabeça etc., gerados pelos usuários, e que podem ser acessados por meio de um navegador da internet ou do aplicativo Kahoot! para dispositivos móveis. O quiz se trata de um jogo no qual os participantes devem tentar responder corretamente as questões que lhes são direcionadas(Houaiss e Villar, 2015). A escolha pela opção do jogo quiz do Kahoot! se deve aos resultados de estudos em diferentes áreas do conhecimento, que demonstram sua eficácia como recurso pedagógico e como fator de motivação para os alunos, bem como sua colaboração no processo de aprendizagem(Araújo et al., 2011; Sales, Leite e Vasconselos, 2014).

fundamentalmente diferente, envolvendo conhecimentos, habilidades e competências específicos, que implicam formas de aprendizagem diferenciadas e, consequentemente, procedimentos diferenciados de ensino."(Soares, 2004). 


\section{Fundamentação teórica}

A origem do termo "gamification" (em português, gamificação) é atribuída ao programador britânico Nick Pelling(Pelling, 2011). Ainda que o termo possa remeter a jogo, a gamificação, na realidade, não consiste em apenas utilizar elementos de jogos em contextos de não-jogo, mas no "uso de mecânicas, estéticas e pensamentos dos games para engajar pessoas, motivar a ação, promover a aprendizagem e resolver problemas"(Fardo, 2013). Dentre os diversos modelos apresentados pela literatura de elementos de jogos, atualmente, o mais aceitado e difundido no contexto de gamificação foi desenvolvido naUniversityofPennsylvania, por (Werbach e Hunter, 2012).

Nesse modelo, as dinâmicas correspondem a "aspetos gerais que determinam como os usuários irão progredir no ambiente gamificado", conhecidas como a gramática da jogabilidade (restrições, emoções, narrativa, progressão, relacionamentos); já as mecânicas são "elementos que promovem (motivam) a ação do usuário para progredir no ambiente gamificado", conhecidas como os verbos da jogabilidade (sorte, cooperação, competição, feedback, aquisição de recursos, recompensas, transações, turnos, estado de vitória); por fim, os componentes são "instâncias específicas das mecânicas e dinâmicas no ambiente gamificado", conhecidos como nomes da jogabilidade - conquistas, avatares, emblemas, coleções, combate, desbloqueio de conteúdo, presentes, placares, níveis, pontos.

Outro conceito fundamental, relacionado à gamificação, baseia-se no que ficou conhecido como flow, em português, estado de fluxo(Csikszentmihalyi, 2008). De acordo com as pesquisas de Csikszentmihalyi, algumas atividades promovem um estado psíquico em que as pessoas que a desempenham estão tão envolvidas em sua realização que nada pode desviar sua atenção. Esse estado se caracteriza por oito componentes: (1) objetivos claros, (2) atenção focada, (3) perda da autoconsciência, (4) sensação de tempo distorcida, (5) senso de feedback imediato e direto, (6) percepção de equilíbrio entre nível de habilidade e desafio; (7) senso de controle de seus atos e (8) motivação intrínseca, a atividade como objetivo por si.

Estudos têm mostrado que a relação entre a motivação e o aprendizado tende a ser bem-sucedida em contextos educacionais que utilizam a gamificação justamente por seus elementos constitutivos - como o feedback constante, a superação, o estado de fluxo etc. (Crespo e Rozenfeld, 2019; Csikszentmihalyi, 2014).

\section{Trabalhos relacionados}

Diversas pesquisas têm mostrado que a aplicação da gamificação em contextos educacionais acarreta resultados positivos(Borges et al., 2013; Hamari, Koivisto e Sarsa, 2014). Um estudo desenvolvido por(Ye, Watthanapas e Wu, 2020), com objetivo de identificar os benefícios da aprendizagem baseada em jogos, mostrou não haver correlação entre o desempenho no aprendizado e a tensão no jogo, sendo assim a tensão não necessariamente afeta a eficiência do aprendizado com base em jogos. Os participantes da pesquisa eram estudantes de duas universidades no norte de Taiwan, que fizeram cursos de língua e cultura tailandesa, com oito semanas de duração. Esses estudantes foram convidados a jogar por 15 minutos o Kahoot! como revisão do conteúdo da semana anterior e a preencherem um questionário na oitava semana. $\mathrm{O}$ número total de participantes válidos foi de 103, incluindo homens $(29,8 \%)$ e mulheres $(70,2 \%)$.

Outra pesquisa, realizada com o Kahoot! em uma turma de graduação de Letras (Alemão) em uma universidade pública do interior paulista. Crespo e Rozenfeld (2019)analisaramo elementode jogo"desafio" nas atividades com a plataforma. Foram coletados dados durante dois meses, em oito turmas da disciplina Língua Alemã I, II, III 
e IV, nos turnos diurno e noturno. No entanto, na pesquisa, foram analisados os dados apenas das aulas ministradas nas disciplinas Língua Alemã I e IV (diurno e noturno), considerada uma amostra representativa dentro do contexto estudado. Concluiu-se que desafios "equilibrados" no Kahoot! podem se constituir em uma alternativa como uma tarefa instigante frente ao ensino convencional(Crespo e Rozenfeld, 2019). Além disso, tais desafios podem contribuir para os elementos do estado de fluxo, como a concentração e o envolvimento.

Outro estudo com foco em elementos do design de jogos foi o desenvolvido na universidade deMichigan. Nesse caso, investigou-se as relações entre a pressão do tempo nos jogos e a autonomia e competência, em dois cenários (com e sem limite de tempo). Os resultados sugerem que pode haver um limite de tempo ideal em que autonomia e competência são potencializadas e, portanto, motivação intrínseca, fluxo, engajamento, desempenho e diversão são promovidos(Yildirim, 2016). O experimento foi conduzido em três etapas: (i) um pré-questionário; (ii) uma sessão (dados demográficos e jogo) antes do laboratório e uma sessão de jogo, e (iii) uma póspesquisa com sessão no laboratório. Os participantes foram divididos em dois grupos (controle e experimental) e foram testados usando duas versões diferentes do jogo (sem e com limite de tempo). Estudantes de graduação e pós-graduação (69 homens, 37 mulheres) da MiddleEastTechnicalUniversity, na Índia, participaram do experimento. Nenhuma diferença significativa na experiência do fluxo foi revelada.

\section{Metodologia}

Nesta seção, apresentamos os procedimentos metodológicos que delinearam este estudo. Ele foi conduzido como um experimento, seguindo os procedimentos delineados em (Gil 2002), no contexto de um cenário real, com o objetivo de caracterizar os efeitos da dinâmica de jogo do "restrição de tempo" (objeto de estudo) na realização da utilização doquiz da plataforma Kahoot! no ensino de literatura.

\subsection{Formulação de hipóteses e variáveis}

Para avaliar os efeitos na "experiência de fluxo" e "aprendizagem" no cenário "com restrição" e "sem restrição" de tempo limite, formulamos as seguintes hipóteses:

- Hipótese Nula $\left(\mathrm{H} 1_{\text {null }}\right)$ : não há diferença significativa na experiência de fluxo para os participantes nos cenários "com restrição" e "sem restrição" de tempo limite em atividades quizzes no ensino de literatura.

- Hipótese Nula $\left(\mathrm{H} 2_{\text {null }}\right)$ : não há diferença significativa na aprendizagem de literatura para os participantes nos cenários "com restrição" e "sem restrição" de tempo limite em atividades de tipo quizzes.

Além da variável independente, tipo de cenário - "com restrição" e "sem restrição" de tempo limite. As variáveis dependentesdas hipóteses foram a "experiência de fluxo" e suas nove dimensõese a "aprendizagem" definida como as "notas obtidas por meio de teste após participar das atividades" (nota.pos) e a "diferença entre a nota obtida após participar das atividades e a nota obtida antes das atividades" (dif.nota).

\subsection{Design do experimento e seleção de participantes}

O estudo empírico foi conduzido como um design de um fator (experiência de fluxo e aprendizado) e dois tratamentos: "com restrição" e "sem restrição" de tempo para completar atividades de tipo quiz no Kahoot!

Para a realização deste estudo, foi empregada a técnica de amostragem aleatória de conveniência. Assim, 50 alunos de duas turmas do $1^{\circ}$ ano do Ensino Médio, de uma escola particular da cidade de Resende, Rio de Janeiro, foram convidados a participardo 
experimento $^{2}$. Por serem turmas regulares da escola, a aleatoriedade de seleção de sujeitos se ateve somente à decisão sobre as turmas escolhidas para participar do cenário "com restrição" e "sem restrição" de tempo limite para completar atividades de tipo quizzes no ensino de literatura.

\subsection{Instrumentos e materiais}

No presente estudo, utilizamos a atividadequiz da plataforma Kahoot! com pontos e tabelas de rankings como dinâmicas de progressão e relacionamento (Figura 1 - lado esquerdo). Após efetuada uma resposta por estudante, sempre é aplicada a mecânica dejogofeedback automático, indicando a resposta correta na corverde e as respostas erradas com cor vermelha (Figura 1 - lado direito). Após, o feedback o estudante pode para a próxima pergunta,não havendo possibilidade de efetuaroutra tentativa.

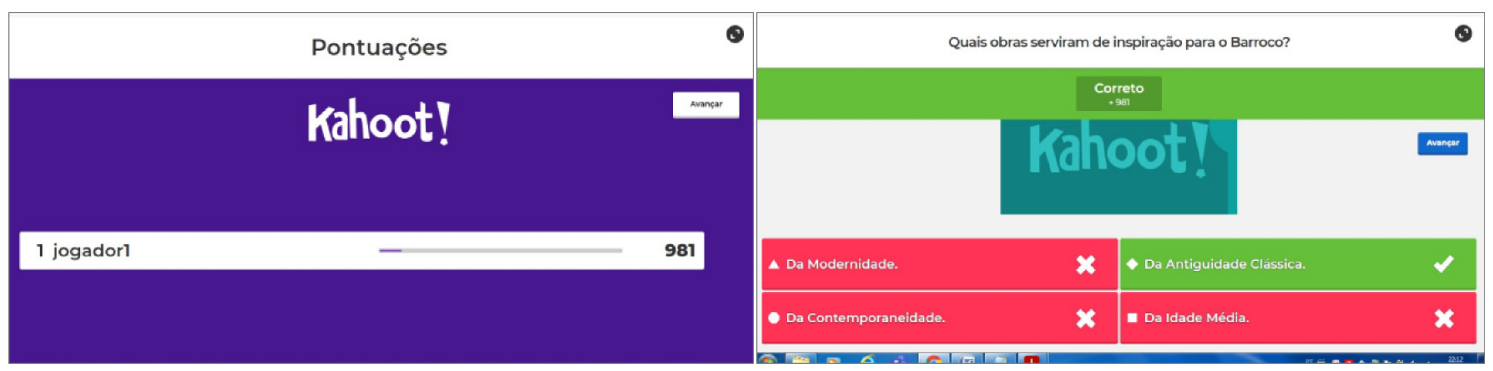

Figura 1 - Dinâmicas de jogos na atividade Quizna plataforma Kahoo!

$\mathrm{Na}$ versão adotada para o cenário "com restrição" de tempo limite (Figura 2 lado esquerdo), o usuário é obrigado a "responder à questão no tempo limite estipulado por um cronômetro de tempo fixo em 150 segundos.Na versão "semrestrição"(Figura 2B), note-se que, na parte esquerda não há um cronômetro para controlar o tempo que resta ao jogador para responder à questão.

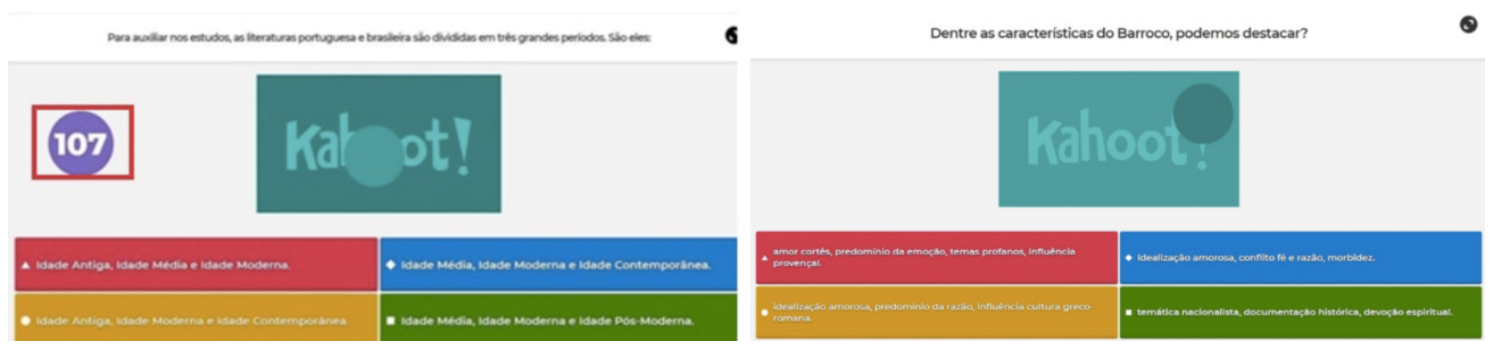

Figura 2 - Telas das atividades em Kahoot! - "com restrição" de tempo limite (tela do loado esquerda) e "sem restrição" de tempo limite (lado esquerdo)

Para medir a experiência de fluxo, foi empregada uma versão adaptada em português brasileiro, do "FlowStateScale"(FSS)(Bittencourt et al., 2021), para indicar a avaliação do estado de fluxo dos participantes de uma atividade que acabam de participar. Para mensurar a aprendizagem, foram empregados dois testes (pré e pósexperimento). $\mathrm{O}$ primeiro teste continha 10 questões objetivas, que foram disponibilizadas por meio da plataforma Geekie, já o segundo, 10 questões objetivas, foram disponibilizadas no Google Forms. As questões foram elaboradas a partir do

\footnotetext{
${ }^{2} \mathrm{Na}$ ocasião, a escola possuía quatro turmas de $1^{\circ}$ ano de Ensino Médio. Cada uma era composta de uma média de 25 a 30 alunos.
} 
conteúdo do movimento literário conhecido como Barroco, previsto na matriz curricular da disciplina Literatura do $1^{\circ}$ ano do Ensino Médio, seguindo as Diretrizes Curriculares do MEC(MEC, 2016).Como a disciplina Literatura não constava do currículo escolar dos alunos até então, já era previsto que eles não tivessem nenhum conhecimento do movimento literário a ser estudado. Então, no pré-teste, foram utilizadas questões do banco de questões da plataforma Geekie. No pós-teste, as questões foram elaboradas pela professora para evitar o compartilhamento de respostas entre os alunos e, consequentemente, ocasionar no comprometimento dos resultados desta pesquisa.Ambas questões foram de multiplica escolha com apenas uma alternativa correta.

\subsection{Procedimento de Coleta de Dados}

Nesta investigação, inicialmente, foi solicitado aos alunos que respondessemopré-teste de dez questões objetivas na plataforma Geekie. Na ocasião, 30 alunos participaram dessa etapa. Então, os grupos tiveram aulas expositivas dialogadas e, posteriormente, foi aplicado o quiz com o Kahoot! (uma turma participou do cenário sem restrição de tempo limite e a outra com a restrição de tempo). Após a aplicação do quiz, foi solicitado, mais uma vez, que os alunos respondessem a outros dois questionários: (i) para medir o fluxo de experiência, eles responderam a FSS, e (ii) para identificaro aprendido, o pós-teste de dez questões objetivas na plataforma Google-form. Dessa vez, 11 participantes responderam ao FSS, e 13, ao questionário de pós-teste.

\section{Resultados e análises de dados}

Devido ao tamanho das amostras de dados obtidas para testar a hipóteses H1, n=11 participantes (6 no cenário com restrição e 5 no cenário sem restrição) e ao tamanho da amostra para testar a hipótese $\mathrm{H} 2, \mathrm{n}=13$ participantes ( 8 no cenário com restrição e 5 no cenário sem restrição), empregamos testes não paramétricos nas análises.

\subsection{Experiência de fluxo (hipóteses H1)}

Os testes de Wilcoxon's Mann-Whitney foram efetuados para comparar as medianas das variáveis dependentes "fluxo", "dimensão 1" (balanço de desafio/habilidade), "dimensão 2" (fusão ação e atenção), "dimensão 3" (objetivos claros), "dimensão 4" (feedback), "dimensão 5" (concentração), "dimensão 6" (controle), "dimensão 7" (perda da autoconsciência), "dimensão 8" (transformação do tempo), "dimensão 9" (experiência autotélica). Os resultados dos testes são apresentados na Tabela 1para as condições: cenário "com restrição" (condição 1) e "sem restrição" (condição 2) de tempo para resolver o quiz de literatura no Kahoot!

Tabela 1. Estatística descritiva e resultado do teste de hipóteses H1

\begin{tabular}{|c|c|c|c|c|c|c|c|c|c|}
\hline Variável & Cenário & $\mathbf{N}$ & Mdn & $\mathbf{M}$ & IQR & DP & W & $\mathbf{r}$ & p \\
\hline fluxo & om restrição & 6 & 3.264 & 3.218 & 0.326 & 0.297 & \multirow{2}{*}{12.00} & \multirow[b]{2}{*}{0.165} & \multirow[b]{2}{*}{0.662} \\
\hline fluxo & sem re & 5 & 3.306 & 3.3 & .306 & & & & \\
\hline imensao1 & $\operatorname{com~r}$ & 6 & & 708 & & & \multirow{2}{*}{20.00} & \multirow{2}{*}{0.279} & \multirow{2}{*}{0.405} \\
\hline dimensao1 & sem 1 & 5 & 3.500 & 3.450 & 0.000 & & & & \\
\hline dimensao2 & com & 6 & 2.8 & 3.0 & & & \multirow[b]{2}{*}{12.00} & \multirow[b]{2}{*}{0.169} & \multirow[b]{2}{*}{0.640} \\
\hline dimensao2 & sem $r$ & 5 & 3.250 & 3.250 & 1.000 & 0.791 & & & \\
\hline & com & 6 & 3.1 & 3.0 & 0.250 & & \multirow{2}{*}{14.00} & \multirow{2}{*}{0.058} & \multirow[b]{2}{*}{0.923} \\
\hline dimensao3 & sem r & 5 & 3.250 & 3.100 & 0.500 & & & & \\
\hline dimensao4 & com restrição & 6 & 2.625 & 2.917 & 0.812 & 0.563 & & & \\
\hline
\end{tabular}




\begin{tabular}{|c|c|c|c|c|c|c|c|c|c|}
\hline dimensao4 & sem restrição & 5 & 3.000 & 2.900 & 0.250 & 0.137 & & & \\
\hline dimensao5 & com restrição & 6 & 3.375 & 3.375 & 0.625 & 0.468 & \multirow{2}{*}{10.00} & \multirow{2}{*}{0.282} & \multirow{2}{*}{0.399} \\
\hline dimensao5 & sem restrição & 5 & 4.000 & 3.600 & .500 & 0.652 & & & \\
\hline dime & com r & 6 & 3.125 & 3.083 & 250 & & \multirow{2}{*}{8.50} & \multirow{2}{*}{0.366} & \multirow{2}{*}{0.262} \\
\hline dime & sem r & 5 & 3.500 & 3.400 & 0.250 & 0.627 & & & \\
\hline dime & com & 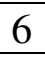 & 3.000 & 3.083 & .938 & & \multirow[b]{2}{*}{11.50} & \multirow[b]{2}{*}{0.194} & \multirow[b]{2}{*}{0.582} \\
\hline dime & sem r & 5 & 3.500 & 3.350 & 0.500 & 0.675 & & & \\
\hline dime & com & & 3.625 & 3.500 & 0.438 & & \multirow[b]{2}{*}{17.50} & \multirow[b]{2}{*}{0.142} & \multirow[b]{2}{*}{0.707} \\
\hline dime & sem restrição & 5 & 3.500 & 3.400 & 0.750 & 0.379 & & & \\
\hline dimer & com restrição & 6 & 3.125 & 3.208 & 0.438 & 10 & \multirow[b]{2}{*}{11.00} & \multirow[b]{2}{*}{0.228} & \multirow[b]{2}{*}{0.508} \\
\hline dimensao9 & sem restrição & 5 & 3.500 & 3.350 & 0.250 & 0.220 & & & \\
\hline
\end{tabular}

$\mathrm{N}$ : número de participantes, Mdn: mediana, M: média, IQR: amplitude interquartil, DP: desvio padrão, W: valor estatístico, r:tamanho de efeito (rank), p: valor-pde significância

De acordo com os resultados obtidos, não houve diferenças estatísticas significativas em nenhuma das variáveis dependentes. Assim, a hipótese nula $\left(\mathrm{H} 1_{\text {null }}\right)$ não é rejeitada. Esses resultados sugerem que o elemento de design de jogo "restrição de tempo para completar uma atividade" não provocou diferenças significativas nos efeitos na experiência de fluxo dos participantes.

\subsection{Aprendizagem (Hipóteses H2)}

Os testes de Wilcoxon's Mann-Whitney foram efetuados para comparar as medianas das variáveis dependentes "nota.pos" (notas no teste após as atividades) e "dif.nota" (diferença entre as notas no teste após e antes das atividades). Os resultados são apresentados na Tabela 2para as condições: cenário "com restrição" (condição 1) e "sem restrição" (condição 2) de tempo para resolver o quiz de literatura no Kahoo!.

Tabela 2. Estatística descritiva e resultado do teste de hipóteses $\mathrm{H} 2$

\begin{tabular}{|c|c|c|c|c|c|c|c|c|c|}
\hline Variável & Cenário & $\mathbf{N}$ & Mdn & $\mathbf{M}$ & IQR & DP & $\mathbf{W}$ & $\mathbf{r}$ & p \\
\hline nota & com restrição & 8 & 93.0 & 92.125 & 10.00 & 6.600 & \multirow[b]{2}{*}{08.0} & \multirow[b]{2}{*}{0.493} & \multirow[b]{2}{*}{0.044} \\
\hline & sem & 5 & & & & & & & \\
\hline dif.nc & com re & 8 & 21.5 & 20.875 & 22.25 & 21. & \multirow[b]{2}{*}{16.5} & \multirow[b]{2}{*}{0.145} & \multirow[b]{2}{*}{0.328} \\
\hline dif.nota & sem restrição & 5 & 20.0 & 27.200 & 8.00 & 12.133 & & & \\
\hline
\end{tabular}

$\mathrm{N}$ : número de participantes, Mdn: mediana, M: média, IQR: amplitude interquartil, DP: desvio padrão, W: valor estatístico, r: tamanho de efeito (rank), p: valor-p de significância

De acordo com os resultados obtidos, para a variável dependente "nota.pos", houve uma diferença significativa, entre o cenário "com restrição" (Mdn=93 and $\mathrm{IQR}=10)$ e o cenário "sem restrição" ( $\mathrm{Mdn}=98$ and $\mathrm{IQR}=2)$ com $\mathrm{W}=8, \mathrm{p}=0.044$ e tamanho de efeito de $\mathrm{r}=0.49$ (moderate). Os resultados sugerem que, no ensino de literatura para estudantes de ensino médio, as notas dos alunos que participaram em atividades de tipo quiz "sem restrição" de tempo limite para completar as atividades são melhores que as notas dos alunos que participaram em cenários "com restrição" de tempo limite.

\section{Discussão, ameaças e limitações do estudo}

Como no estudo apresentado por Yeet al (2020), nosso estudo investigou se a dinâmica de restrição de tempo causaria algum efeito na experiência de fluxo e aprendizagem de estudantes em atividades de tipo quiz. Empregando a mesma plataforma Kahoot! 
utilizada no estudo citado, constatou-se que não houve nenhuma diferença significativa na experiência de fluxo. Estudantes que participaram do cenário sem restriçãoobtiveram notasligeiramente maiores nos resultados do pós-teste, no entanto,cabe esclarecer que,o estudo prévio foi realizado com participantes de diferente faixa etária, isso podem ter causado efeito diferentee precisa ser estudado no futuro.

Entende-se que os estudantes que participaram do cenário "sem restrição" tiveram mais tempo para ler os enunciados, refletir acerca de suas respostas nos quizzes, e até mesmo para consultar outras fontes, o que não foi possível aos participantes do cenário "com restrição" de tempo. A realização dessas atividades pode ser uma explicação plausível por que estudantes que fizeram quiz sem restrição do tempo tiveram melhor desempenho no pós-teste do que estudantes com restrição de texto.

Adicionar tempo limite na realização de uma atividade incrementa o seu nível de desafio. Crespo e Rozenfeld (2019)apontam o elemento desafio como importantes para a concentração e o envolvimento. Em nosso estudo, pode ser inferido isso como uma explicação plausível de porque no cenário com restrição os participantes tiveram menor concentração (dimensão 5) ou envolvimento (dimensões 6, 7 e 8) quando comparado com o cenário sem restrição. Maior desafio pode ter levado a sentir pressão/tensão causando efeito negativos nesses construtos psicológicos. No entanto, esses efeitos não são significativos em ambos os grupos, reforçando assim a ideia de que existe um "tempo limite ideal." Como foi apontado por Yıldırım (2016),quando a restrição de tempo está do"tempo limite ideal" pode promover o fluxo e aprendizado, quando não, ela pode causar efeito contrário, o que pode ter acontecido em nosso estudo.

Em relação à validade dos resultados da presente pesquisa, a falta de preenchimento de todos os questionários por parte dos estudantes impossibilitou a aplicação de testes paramétricos, bem como para identificar os perfis dos jogadores. Aliado a isso, a amostra foi composta,em sua maioria, por participantes do sexo feminino, o que impossibilitou fazer análises estadísticos no sentido de estabelecer diferenças de efeitos de acordo com o gênero dos participantes.

Em virtude do ensino remoto oferecido noperíodo de isolamento causado pela pandemia de Covid-19, durante a aplicação do experimento, o acesso de todas as atividades de casa, sem a supervisão do professor, permite trocas entre os estudantes, inclusive consultas de material externo durante as avaliaçõesque foram aplicadas no pré-teste e pós-teste. No que se refere às limitações para a coleta de dados durante o estudo, o pesquisador observou que o acesso dos estudantes à internet foi precário, realizado por aparelhos de celular em sua maioria (e não computadores). Isso pode ter refletidona aderência em participar no estudo e no preenchimento dos questionários, assim como na participação dos estudantes durante o estudo.

\section{Conclusão e trabalhos futuros}

Este estudo teve como objetivo compreender os efeitos na experiência de fluxo e de aprendizagem do elemento de design de jogo "restrição de tempo para completar uma atividade" em quizzes de literatura para alunos do ensino médio. A investigação visa contribuir no entendimento de como um elemento degamificaçãoafeta o engajamento dos estudantes em atividades educacionais.

Partindo desses pressupostos, percebeu-se que, mesmo que diferentes cenários com ou sem restrição de tempo não resulte em diferenças significativas no fluxo de experiência dos alunos, a restrição pode influenciar no aprendizado. Considerando as dificuldades inerentes ao processo de leitura e interpretação de texto que a maioria dos alunos apresentam em testes padronizados, é possível depreender que os estudantes que participaram do experimento no cenário "sem restrição de tempo" tiveram como 
vantagem a possibilidade de participar do cenário gamificado no seu próprio ritmo, o que pode justificar o maior desempenho no pós-teste aplicado.

Dessa forma, na utilização da plataformaKahoot! no ensino de literatura deve-se considerar que, dependendo do limite do tempo, essa restrição pode desfavorecer o aprendizado dos alunos. Em trabalhos futuros, sugere-se que seja investigado o que pode ser considerado como o "tempo ideal", apontado nos estudos de Yıldırım (2016), para potencializar os resultados em atividades com a utilização da gamificação.

\section{Referências}

ARAÚJO, G. H. M.; SIlva, A. S. C.; CARVAlHO, L. A. S.; SILVA, J. C.; RODRIGUES, C.; OLIVEIRA, G. F. O quiz como recurso didático no processo ensinoaprendizagem em genética. 63 $^{\text {a }}$ Reunião Anual da SBPC, n. 2176-1221, 2011.

BITTENCOURT, I. I.; FREIRES, L.; LU, Y.; CHALLCO, G. C.; FERNANDES, S.; COELHO, J.; COSTA, J.; PIAN, Y.; MARINHO, A.; ISOTANI, S. Validation and psychometric properties of the Brazilian-Portuguese dispositional flow scale 2 (DFSBR). Plos one, v. 16, n. 7, p. e0253044, 2021.

BORGES, S. DE S.; REIS, H. M.; DURELLI, V. H.; BITTENCOURT, I. I.; JAQUES, P. A.; ISOTANI, S. Gamificação aplicada à educação: um mapeamento sistemáticoBrazilian Symposium on Computers in Education (Simpósio Brasileiro de Informática na Educação-SBIE). Anais...2013

CHALlCO, G. C.; ANDRADE, F. R. H.; BORGES, S. S.; BITTENCOURT, I. I.; ISOTANI, S. Toward a unified modeling of learner's growth process and flow theory. Educational Technology and Society, v. 19, n. 2, p. 215-227, 2016.

COSSON, R. Letramento Literário. Teoria e Prática. São Paulo: Contexto, 2006.

CRESPO, A. H.; ROZENFELD, C. C. DE F. O Estado de Fluxo (Flow) na aprendizagem de alemão durante o uso da ferramenta Kahoot!: um olhar sobre o nível de desafio. Revista Linguagem \& Ensino, v. 22, n. 4, p. 1063-1088, 2019.

CSIKSZENTMIHALYI, M. Flow: The Psychology of Optimal Experience. 1ST edition ed. New York: Harper Perennial Modern Classics, 2008.

Learning, "Flow," and HappinessApplications of Flow in Human Development and Education. Anais...Springer, 1 jan. 2014Disponível em: <http://link.springer.com/chapter/10.1007/978-94-017-9094-9_7>. Acesso em: 16 jan. 2015

DICHEVA, D.; DICHEV, C.; AGRE, G.; ANGELOVA, G. Gamification in education: A systematic mapping study. Educational Technology and Society, v. 18, p. 75-88, 2015.

FAIELLA, F.; RICCIARDI, M. Gamification and learning: A review of issues and research. Journal of E-Learning and Knowledge Society, v. 11, n. 3, p. 13-21, 2015.

FARDO, M. L. A Gamificação Aplicada em Ambientes de Aprendizagem. RENOTE, v. 11, n. 1, 30 jul. 2013.

FORMIGA, G. M.; INÁCIO, F. A. Literatura no ensino médio: reflexões e proposta metodológica. Revista Brasileira de Literatura Comparada, v. 15, n. 22, p. 179-198, 2017. 
HAMARI, J.; KOIVISTO, J.; SARSA, H. Does gamification work? - A literature review of empirical studies on gamificationProc. Annu. Hawaii Int. Conf. Syst. Sci. Anais... In: 47TH HAWAII INTERNATIONAL CONFERENCE ON SYSTEM SCIENCES, HICSS 2014. IEEE Computer Society, 2014. Acesso em: 6 jan. 2014

HOUAISS, A.; VILLAR, M. Grande dicionário Houaiss da língua portuguesa. [s.1.] Círculo de Leitores, 2015.

MEC. Base Nacional Comum Curricular. A base. 2016. Disponível em: $<\mathrm{http}$ ://basenacionalcomum.mec.gov.br/a-base $>$.

MORA, A.; RIERA, D.; GONZÁLEZ, C.; ARNEDO-MORENO, J. Gamification: a systematic review of design frameworks. Journal of Computing in Higher Education, v. 29, n. 3, p. 516-548, 2017.

OLIVEIRA, W.; PASTUSHENKO, O.; RODRIGUES, L.; TODA, A. M.; PALOMINO, P. T.; HAMARI, J.; ISOTANI, S. Does gamification affect flow experience? A systematic literature review. arXiv e-prints, p. arXiv-2106, 2021.

PELLING, N. The (short) prehistory of "gamification"...Funding Startups (\& other impossibilities), 2011. Disponível em: $<$ https://nanodome.wordpress.com/2011/08/09/the-short-prehistory-of-gamification/>.

Acesso em: 3 maio. 2021

SALES, G. L.; LEITE, E. A. M.; VASCONSELOS, F. H. L. Quiz online como Suporte à Aprendizagem de Física no Ensino MédioNuevas Ideas en Informática Educativa. In: nuevas ideas em Informática educativa: Memorias del XVI Congreso Internacional de Informática Educativa, Santiago de Chile. Anais...2014

SANTOS, W. O. DOS; BITTENCOURT, I. I.; ISOTANI, S.; DERMEVAL, D.; MARQUES, L. B.; SILVEIRA, I. F. Flow Theory to Promote Learning in Educational Systems: Is it Really Relevant? Revista Brasileira de Informática na Educação, v. 26, n. 02, p. 29, 23 maio 2018.

SILVA, R.; RODRIGUES, R.; LEAL, C. Gamification in management education - A literature mapping. Education and Information Technologies, 26 nov. 2019.

SOARES, M. Letramento e alfabetização: as muitas facetas. Revista Brasileira de Educação, n. 25, p. 5-17, 2004.

WERBACH, K.; HUNTER, D. For the win: How game thinking can revolutionize your business. [s.l.] Wharton Digital Press, 2012.

YE, J.-H.; WATTHANAPAS, N.; WU, Y.-F. Applying Kahoot in Thai Language and Culture Curriculum: Analysis of the Relationship among Online Cognitive Failure, Flow Experience, Gameplay Anxiety and Learning Performance. International Journal of Information and Education Technology, v. 10, n. 8, p. 563-572, 2020.

YILDIRIM, I. G. Time Pressure as Video Game Design Element and Basic Need SatisfactionProceedings of the 2016 CHI Conference Extended Abstracts on Human Factors in Computing Systems. Anais...: CHI EA '16.New York, NY, USA: Association for Computing Machinery, 2016Disponível em: <https://doi.org/10.1145/2851581.2892298>. Acesso em: 3 maio. 2021. 